\title{
Radio continuum and radio recombination line observations of the Galactic center lobe
}

\author{
Halca Nagoshi ${ }^{1}$, Kenta Fujisawa ${ }^{2}$ and Yuzo Kubose ${ }^{3}$ \\ ${ }^{1}$ Graduate school of Science and Engineering, Yamaguchi University, 1677-1 Yoshida, \\ Yamaguchi, Yamaguchi 753-8512, Japan \\ email: s002wa@yamaguchi-u.ac.jp \\ ${ }^{2}$ Department of Physics, Faculty of Science, Yamaguchi University, 1677-1 Yoshida, \\ Yamaguchi, Yamaguchi 753-8512, Japan \\ email: kenta@yamaguchi-u.ac.jp \\ ${ }^{3}$ Graduate school of Science and Engineering, Yamaguchi University, 1677-1 Yoshida, \\ Yamaguchi, Yamaguchi 753-8512, Japan, Japan \\ email: t002vc@yamaguchi-u.ac.jp
}

\begin{abstract}
Radio continuum (cont) and radio recombination line (RRL) observations with the Yamaguchi 32-m radio telescope toward the lower part of the Galactic center lobe (GCL) in the Galactic center region are presented. While two ridges of the GCL were seen in both continuum and RRL images, the spatial coverage of the ridges of the continuum and RRL is not coincident. We distinguish the continuum emission of the GCL into thermal and non-thermal emission by assuming an electron temperature of the ionized gas of $4370 \mathrm{~K}$, estimated based on the line width $\left(14.1 \mathrm{~km} \mathrm{~s}^{-1}\right)$. The thermal emission was found to be located inside and surrounded by the non-thermal emission.
\end{abstract}

Keywords. (ISM:) HII regions, radio continuum: ISM, radio lines: ISM

\section{Introduction}

The Galactic center lobe (GCL), discovered in $10 \mathrm{GHz}$ radio continuum observations (Sofue \& Handa 1984), is a one degree tall lobe-like structure located toward the Galactic center. The radio continuum emission of GCL is considered as an overlap of non-thermal and thermal emission, because linear polarization and radio recombination lines were detected (Tsuboi et al. 1986, Law et al. 2009). In order to distinguish these two emission distributions, we have made radio continuum and recombination line observations.

\section{Observation}

The observations were made with the Yamaguchi 32-m radio telescope. The continuum observation was made at $8.3 \mathrm{GHz}$ on 16 and 17, April 2006, with a $1^{\prime}$ sampling grid for a $5^{\circ} \times 5^{\circ}$ area centered on the Galactic center. The H92 $\alpha(8.3 \mathrm{GHz})$ recombination line observation was made in 2011 and 2012 toward the lower part of the GCL $\left(-60^{\prime}<\right.$ $l<32^{\prime}, 6^{\prime}<b<33^{\prime}$ ) with sampling a grid of $4^{\prime}$ by $3^{\prime}$ in longitude and latitude in Galactic coordinates. The velocity resolution was $2.34 \mathrm{~km} \mathrm{~s}^{-1}$, and the detection limit set to $2 \sigma$ was $5.6 \mathrm{mK}$. The raw spectra were fitted with Gaussian functions after baseline subtraction. 

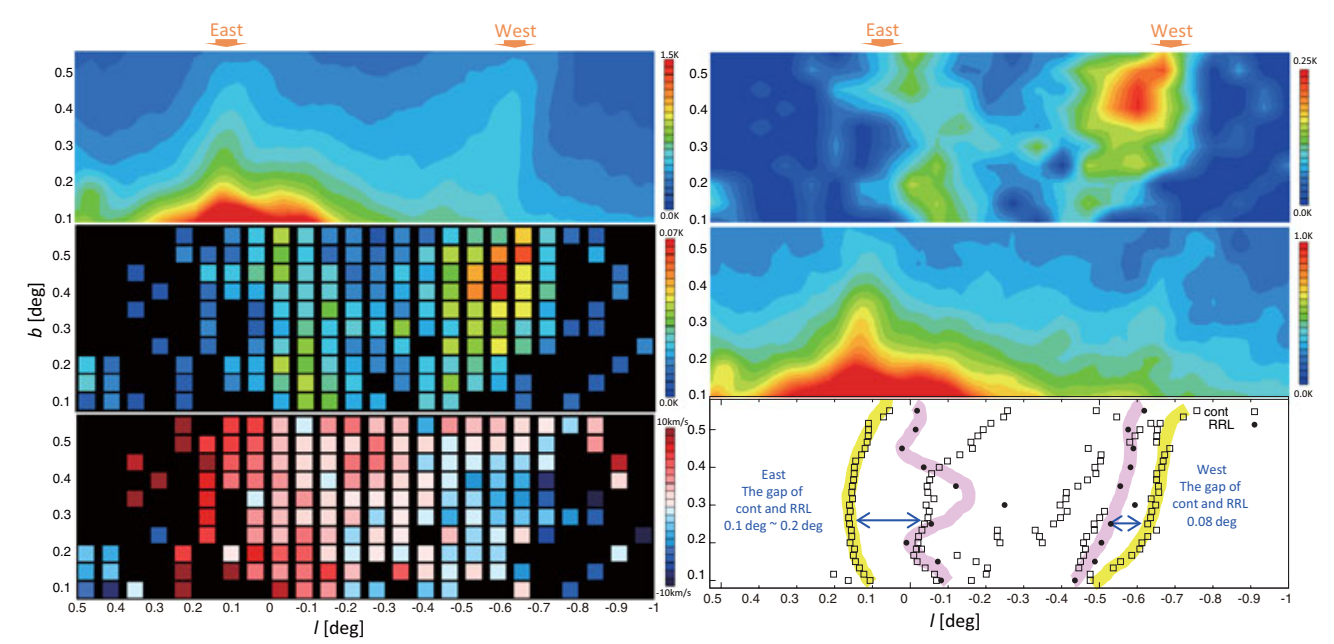

Figure 1. Left: The intensity distribution of continuum (top panel) and RRL (middle panel) for the observation area $\left(-60^{\prime}<l<32^{\prime}, 6^{\prime}<b<33^{\prime}\right)$. The lower panel is the distribution of line-of-sight velocities of RRL. Right: The intensity distribution of thermal emission estimated from RRL which shows the distribution of ionized gas (top panel). The intensity distribution of non-thermal emission (middle panel) is obtained by subtracting the thermal emission from the continuum image. The lower panel is the emission peak plot of continuum and RRL. [A COLOR VERSION IS AVAILABLE ONLINE.]

\section{Result and discussion}

Figure 1 shows the observed intensity distribution of the continuum (top-left) and recombination line (middle-left). The bottom-left panel shows the peak velocity distribution of RRL; a velocity gradient from west to east $\left(-10\right.$ to $\left.+10 \mathrm{~km} \mathrm{~s}^{-1}\right)$ is seen. The average line width (FWHM) is $14.1 \mathrm{~km} \mathrm{~s}^{-1}$ for the strong (>50 mK) RRLs. The electron temperature of thermal plasma derived from the line width is $4370 \mathrm{~K}$, which is consistent with the result obtained by Law et al. (2009) (3960 K, FWHM=13.5 $\left.\mathrm{km} \mathrm{s}^{-1}\right)$.

While two ridges of GCL were seen in both continuum and RRL images, the spatial positions of the ridges of the continuum and RRL are not coincident. We distinguished the continuum emission of GCL into thermal (Figure 1, top-right) and non-thermal emissions (middle-right) by assuming the electron temperature of ionized gas of $4370 \mathrm{~K}$. To compare the distributions of continuum and RRL precisely, the peak positions at each latitude were estimated by multi-component Gaussian fitting. The peak positions of continuum and RRL is shown in Figure 1 bottom-right panel. The eastern ridges of continuum and RRL is separated by $0.1^{\circ}-0.2^{\circ}$, and the separation at the western ridge is $0.08^{\circ}$. The thermal emission is found to be located inside and surrounded by the non-thermal emission.

By assuming a physical depth of $50 \mathrm{pc}$ of the thermal plasma, an electron density of $5.1 \mathrm{~cm}^{-3}$, a mass of ionized gas of $1.0 \times 10^{5} \mathrm{M}_{\odot}$, and a thermal energy of $1.1 \times 10^{50}$ erg were derived. The mass and thermal energy is comparable with those of the outflows in dwarf galaxies (mass $10^{5-6} \mathrm{M}_{\odot}$, energy $10^{50-54} \mathrm{erg}$, Veilleux et al. 2005 ). Since the estimated cooling time of the ionized gas of $5.5 \times 10^{3} \mathrm{yr}$ is much shorter than the timescale of the starburst $\left(10^{5-8} \mathrm{yr}\right)$, the thermal gas of GCL is being heated. 


\section{References}

Sofue, Y. \& Handa, T. 1984, Nature 310, 568

Tsuboi, M., et al. 1986, AJ 92, 818

Law, C. J., Backer, D., Yusef-Zadeh, F., \& Maddalena, R. 2009, ApJ 695, 1070

Veilleux, S., Cecil, G., \& Bland-Hawthorn, J. 2005, ARAA 43, 769 